\title{
Grading Aortic Stenosis With Mean Gradient and Aortic Valve Area: A Comparison Between Preoperative Transthoracic and Precardiopulmonary Bypass Transesophageal Echocardiography
}

\author{
George Whitener, MD,* Joseph Sivak, MD, + Igor Akushevich, PhD, $\neq$ Zainab Samad, MD, MHS, $\uparrow$ and \\ Madhav Swaminathan, MD, MB, BS, FASE*
}

\begin{abstract}
Objective: The authors hypothesized that average precardiopulmonary bypass (pre-CPB) transesophageal echocardiographic (TEE) mean gradient $\left(\mathrm{PG}_{\mathrm{m}}\right.$ ) and aortic valve area (AVA) values would be significantly different from preoperative transthoracic (TTE) values in the same patients and that these changes would affect pre-CPB TEE grading of aortic stenosis (AS).

Design: Retrospective, observational design.

Setting: Single university hospital.

Participants: The study comprised 92 patients who underwent aortic valve replacement with or without coronary artery bypass grafting between 2000 and 2012 at Duke University Hospital and who had $\mathrm{PG}_{\mathrm{m}}$ and AVA values recorded in both pre-CPB TEE and preoperative TTE reporting databases.

Interventions: None.

Measurements and Main Results: PG $_{m}$ with pre-CPB TEE was lower by $6.6 \mathrm{mmHg}$ (95\% confidence interval, -4.0 to $9.3 \mathrm{mmHg} ; \mathrm{p}<0.001$ ), whereas AVA was higher by $0.10 \mathrm{~cm}^{2}$ (95\% confidence interval, 0.04 to $0.15 \mathrm{~cm}^{2} ; \mathrm{p}<0.001$ ), compared with preoperative TTE values. When using $\mathbf{P G}_{\mathrm{m}}$, pre-CPB TEE generated an AS severity 1 grade lower $39.1 \%$
\end{abstract}

$\mathbf{P}$ RECARDIOPULMONARY BYPASS (pre-CPB) transesophageal echocardiography (TEE) is essential for intraoperative assessment and surgical guidance during cardiac surgery. Although many patients come to the operating room with an extensive cardiac workup, pre-CPB TEE aortic valve assessment can affect surgical decision-making based on new findings during planned or emergency surgeries. According to a large retrospective review of 3,835 patients undergoing isolated coronary artery bypass grafting (CABG), $3.3 \%$ of patients had an unplanned aortic or mitral valve procedure added to the surgery based on pre-CPB TEE findings. ${ }^{1}$ Of 1,823 patients

From the *Department of Anesthesiology, Duke University; tDepartment of Cardiology, Duke University Medical Center; and $\ddagger$ Duke University Social Science Research Institute, Duke University, Durham, NC.

The abstract of this article was presented previously, in part, at the 2015 Society of Cardiovascular Anesthesiology National Meeting in Washington, DC (the abstract was selected for poster presentation).

The patients in this study were a subset of patients of another study for a different analysis published in: Whitener $G$, McKenzie J, Akushevich I, et al: Discordance in grading methods of aortic stenosis by precardiopulmonary bypass transesophageal echocardiography. Anesth Analg 122:953-958, 2016

Address reprint requests to George Whitener, MD, Medical University of South Carolina, Department of Anesthesia and Perioperative Medicine, 25 Courtenay Dr, 4210 Ashley River Tower, Charleston, SC 29425. E-mail: whiteneg@musc.edu

(C) 2016 Elsevier Inc. All rights reserved.

1053-0770/2601-0001\$36.00/0

http://dx.doi.org/10.1053/j.jvca.2016.05.012 of the time and revealed no difference $55.4 \%$ of the time compared to preoperative TTE. When using AVA by continuity, pre-CPB TEE generated an AS severity 1 grade lower $14.1 \%$ of the time and revealed no difference $81.5 \%$ of the time compared to preoperative TTE. When using either PGm or AVA, preoperative TTE exhibited moderate or severe AS for all study patients, whereas, pre-CPB TEE demonstrated mild AS in $5.4 \%(n=92)$ of patients.

Conclusions: The authors confirmed their hypothesis that pre-CPB TEE generates different $P_{m}$ and AVA values compared with preoperative TTE. These differences often underestimate AS severity. Hemodynamic standardizations or adjustments of pre-CPB TEE PG $_{m}$ and AVA values may be necessary in anesthetized patients before assigning an AS grade using these parameters.

(c) 2016 Elsevier Inc. All rights reserved.

KEY WORDS: aortic stenosis, aortic valve area, discordance, mean gradient, precardiopulmonary bypass (intraoperative) transesophageal echocardiography

undergoing mitral valve surgery, $1.0 \%$ of patients underwent an unplanned aortic valve procedure based on incidental findings during pre-CPB TEE. ${ }^{1}$ Given that surgical decisions can be based on pre-CPB TEE findings, accurate interpretation of grading parameters during the pre-CPB period is imperative.

The validated mean gradient $\left(\mathrm{PG}_{\mathrm{m}}\right)$ and aortic valve area (AVA) grading cutoffs for aortic stenosis (AS) originate from studies that used transthoracic echocardiography (TTE) in spontaneously ventilating patients without the influence of general endotracheal anesthesia. ${ }^{2-6}$ Thus, anesthesiologists working with pre-CPB TEE must apply cutoffs derived from a different physiologic setting. The misapplication of TTE AS grading cutoffs during pre-CPB TEE could negatively affect patient care if the surgical plan is changed or erroneously unchanged based on pre-CPB TEE findings.

Given these concerns, the authors conducted a retrospective observational study comparing preoperative TTE $\mathrm{PG}_{\mathrm{m}}$ and AVA values with pre-CPB TEE $P_{m}$ and AVA values for patients undergoing aortic valve replacement (AVR) with or without $\mathrm{CABG}$. The authors hypothesized that average pre-CPB TEE PG $_{\mathrm{m}}$ measurements and AVA calculations would be significantly different from preoperative TTE values and that these differences would affect the grading of AS during preCPB TEE.

\section{METHODS}

After obtaining Duke University Institutional Review Board approval, which included a waiver of consent for a retrospective review, the authors obtained data for patients who underwent AVR for AS with or without CABG from January 1, 2000 
through December 31, 2012. Initial patients were identified from the Duke University Department of Anesthesiology perioperative echocardiography database, which includes preCPB TEE reports on all patients undergoing cardiac surgery at Duke University Medical Center during the specified time period.

Comparison reports for the same patients were obtained from the Duke Echocardiography Lab Database, which is a prospectively maintained digital archive of all echocardiograms performed at Duke University Hospital and satellite clinics since 1995. This is linked to a corresponding searchable reporting database populated with the information from the clinical interpretation for each study.

\section{Patient Selection Criteria}

The authors reviewed all reports in the pre-CPB TEE database of patients who underwent AVR for AS with or without CABG from January 1, 2000 to December 31, 2012. Patients were excluded if they underwent emergency surgery or repeat sternotomy. Patients with a reported left ventricular ejection fraction $(\mathrm{LVEF})<55 \%$, severe aortic insufficiency or moderate or severe mitral regurgitation also were excluded. The remaining patients were cross-referenced to the preoperative TTE database to compare $\mathrm{PG}_{\mathrm{m}}$ and AVA values. Patients were included only if a TTE was performed within 6 months before surgery and the $\mathrm{PG}_{\mathrm{m}}$ and AVA values were recorded for both TTE and TEE. If multiple TTE reports were available for the same patient within the 6-month window, the report nearest the date of surgery was used.

\section{Variable Definitions and Echocardiographic Analysis}

$\mathrm{PG}_{\mathrm{m}}$ and AVA values were obtained retrospectively from the clinical reports for each echocardiography examination. At the authors' institution, these values are obtained regularly according to practice guidelines proposed by the American Society of Echocardiography. ${ }^{7} \mathrm{PG}_{\mathrm{m}}$ was obtained by integrating a continuous-wave Doppler tracing of flow across the aortic valve. For pre-CPB TEE, either the deep transgastric aortic valve long-axis view or the transgastric long-axis view was used to align the sample volume as parallel to blood flow as possible. For preoperative TTE, the flow across the aortic valve was examined from multiple windows including apical, suprasternal notch, and right parasternal views, with the highest gradient recorded. Standard, commercially available ultrasound machines recorded mean gradients after manual tracing of the continuous-wave Doppler waveform.

AVA was calculated via the continuity equation ${ }^{3,8}$ for both imaging modalities:

$\operatorname{AVA}\left(\mathrm{cm}^{2}\right)=\frac{\left(\mathrm{CSA}_{\mathrm{LVOT}}\right) \times\left(\mathrm{VTI}_{\mathrm{LVOT}}\right)}{\operatorname{VTI}_{\mathrm{AV}}}$,

where VTI is the velocity time integral.

To determine the cross-sectional area (CSA) of the left ventricular outflow tract (LVOT), the LVOT diameter was obtained using the midesophageal aortic valve long-axis view in pre-CPB TEE and using the parasternal long-axis view in preoperative TTE. LVOT diameters were obtained within 0.5 to $1.0 \mathrm{~cm}$ of the valve orifice at the location of the LVOT VTI measurement, as recommended by European Association of
Echocardiography/American Society of Echocardiography guidelines. ${ }^{9}$ CSA was estimated using the following formula: $C S A=\pi(d / 2)^{2}$, where $d$ is the LVOT diameter. VTIs of the LVOT and AVA were measured via pulsed-wave Doppler and continuous-wave Doppler, respectively. Parameters recorded during pre-CPB TEE were obtained according to institutional protocol during normotension (systolic blood pressure $<140$ $\mathrm{mmHg}$ to $>100 \mathrm{mmHg}$ ) after induction of general anesthesia and before sternotomy. Parameters recorded during preoperative TTE were obtained during the patient's baseline hemodynamics at the time of the study.

All preoperative TTE examinations were performed and reviewed by cardiologists with level III training. All pre-CPB TEE examinations were obtained by or under the supervision of a cardiothoracic anesthesiologist certified by the National Board of Echocardiography (NBE) for special competence in advanced perioperative TEE. Pre-CPB examinations were performed in the context of a robust cardiothoracic anesthesiology fellowship program, which performs more than 1,500 examinations annually. In addition, all images and reports from pre-CPB examinations were read a second time by a separate, NBE-certified echocardiographer offline to ensure the accuracy of the report.

\section{Statistical Analysis}

After obtaining $\mathrm{PG}_{\mathrm{m}}$ and AVA values for all patients meeting inclusion/exclusion criteria, the differences between $\mathrm{PG}_{\mathrm{m}}$ and AVA during pre-CPB TEE versus preoperative TTE were calculated for each patient. Continuous data are represented as mean \pm standard deviation. Mean values for $\mathrm{PG}_{\mathrm{m}}$ and AVA were compared using a paired $t$ test. A p value $<0.05$ was considered significant. Standard deviations were calculated for the overall means of $\mathrm{PG}_{\mathrm{m}}$ and AVA for TTE and pre-CPB TEE, and 95\% confidence intervals (CI) were calculated for the mean differences of $\mathrm{PG}_{\mathrm{m}}$ and AVA between the imaging modalities.

In addition, the authors constructed a table illustrating the number of patients whose AS grade changed between preoperative TTE and pre-CPB TEE when using either $\mathrm{PG}_{\mathrm{m}}$ or AVA. Grading demarcations were taken from American College of Cardiology/American Heart Association and American Society of Echocardiography guideline recommendations. ${ }^{2,9}$ Grading cutoffs used for $\mathrm{PG}_{\mathrm{m}}$ were $\geq 40 \mathrm{mmHg}, 40 \mathrm{mmHg}$ to $\geq 20$ $\mathrm{mmHg}$, and $<20 \mathrm{mmHg}$ for severe, moderate, and mild AS, respectively. Meanwhile, grading cutoffs for AVA were $\leq 1.0$ $\mathrm{cm}^{2}, 1.0$ to $\leq 1.5 \mathrm{~cm}^{2}$, and $<1.5 \mathrm{~cm}^{2}$ for severe, moderate, and mild AS, respectively; $p$ values for comparing the percentage of patients with a grade change in either direction compared with no change were calculated using 2-sided exact tests for binomial proportions.

\section{RESULTS}

A total of 277 patients met surgical inclusion criteria. Of those patients, 92 met inclusion criteria for this study, with an available preoperative TTE report for comparison (Fig 1). Patient characteristics for the final study population are summarized in Table 1. The comparative echocardiography results are summarized in Table 2. The mean $\mathrm{PG}_{\mathrm{m}}$ using 


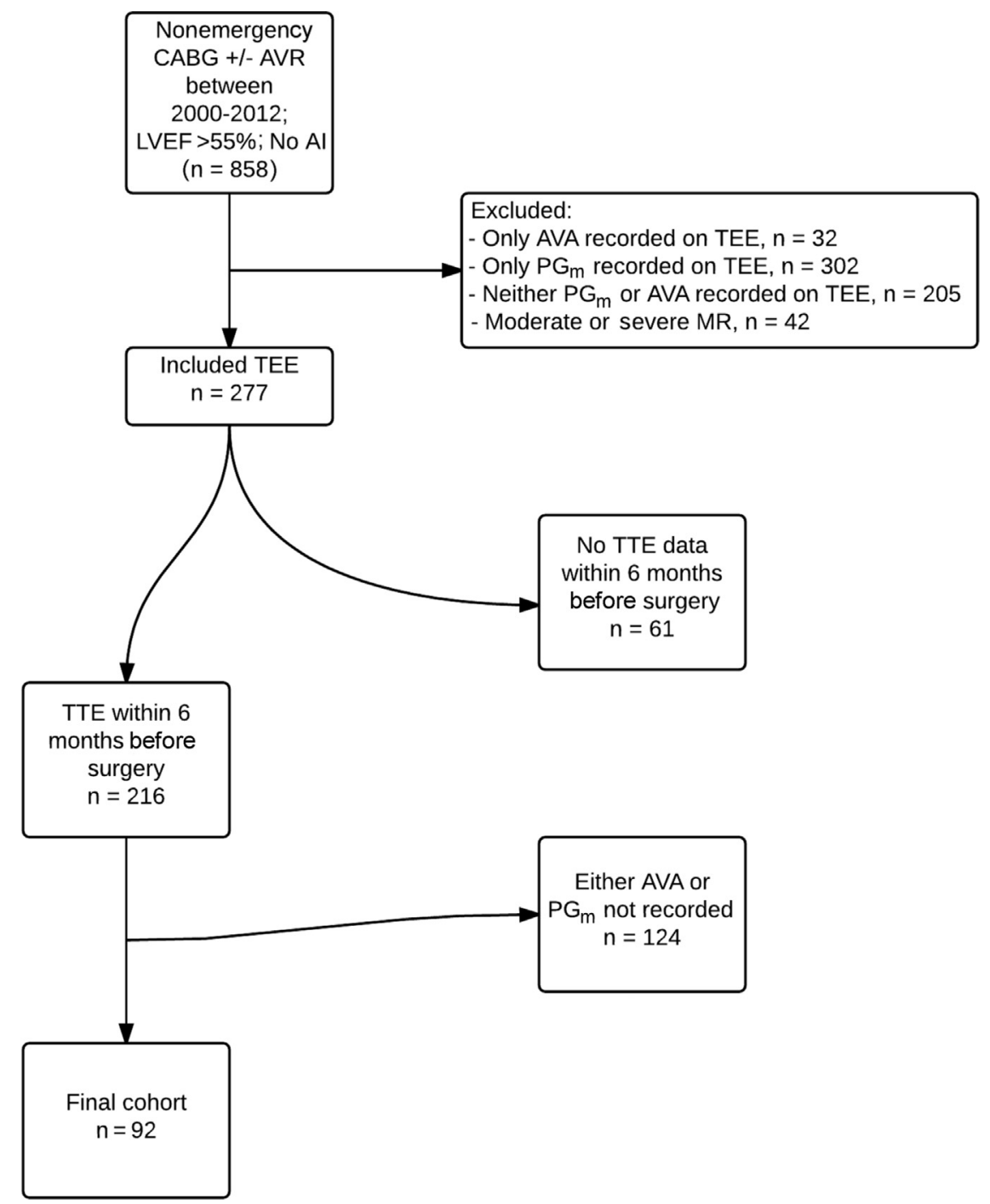

Fig 1. Flow diagram illustrating selection of the study population. Al, aortic insufficiency, AVA, aortic valve area; AVR, aortic valve replacement; CABG, coronary artery bypass grafting; LVEF, left ventricular ejection fraction; $M R$, mitral regurgitation; $\mathbf{P G}_{m}$, mean pressure gradient; TEE, transesophageal echocardiography; TTE, transthoracic echocardiography.

preoperative TTE was $46.0 \pm 14.3 \mathrm{mmHg}$, whereas the mean $\mathrm{PG}_{\mathrm{m}}$ using pre-CPB TTE was $39.4 \pm 14.1 \mathrm{mmHg}$. Thus, the average $\mathrm{PG}_{\mathrm{m}}$ using pre-CPB TEE was significantly lower, by $6.6 \mathrm{~mm} \mathrm{Hg}(95 \% \mathrm{CI},-4.0$ to $-9.3 \mathrm{~mm} \mathrm{Hg} ; \mathrm{p}<0.001)$, compared with the preoperative TTE value. Meanwhile, the average AVA during preoperative TTE was $0.73 \pm 0.24 \mathrm{~cm}^{2}$, whereas the average AVA during pre-CPB TEE was $0.83 \pm 0.31$. Therefore, the average AVA using pre-CPB TEE was significantly higher, by $0.10 \mathrm{~cm}^{2}$ (95\% CI, 0.04$\left.0.15 \mathrm{~cm}^{2} ; \mathrm{p}<0.001\right)$, compared with the preoperative TTE value.

Table 3 summarizes how these differences affected AS severity grading between preoperative TTE and pre-CPB TEE. When using $\mathrm{PG}_{\mathrm{m}}$, pre-CPB TEE generated an AS severity 1 grade lower than preoperative TTE $39.1 \%$ of the time, no difference $55.4 \%$ of the time, and 1 grade higher $5.4 \%$ of the time. Any change in severity was noted $44.5 \%$ of the time for $\mathrm{PG}_{\mathrm{m}}$. Alternatively, when using AVA, pre-CPB TEE generated an AS severity 2 grades lower than preoperative TTE $1.1 \%$ of the time, 1 grade lower $14.1 \%$ of the time, no difference $81.5 \%$ of the time, and 1 grade higher $4.3 \%$ of the time. Any change in severity was noted $18.5 \%$ of the time for AVA. Finally, $5.4 \%(n=5 / 92)$ of patients who experienced mild AS as classified by at least 1 grading parameter during pre-CPB TEE (2 patients were classified as experiencing mild AS using $\mathrm{PG}_{\mathrm{m}}$ 2 patients were classified as experiencing mild AS using AVA, and 1 patient was classified as experiencing mild AS using both parameters) experienced at least moderate AS or greater using both grading parameters during preoperative TTE.

\section{DISCUSSION}

The main findings of this study can be summarized as follows: (1) $\mathrm{PG}_{\mathrm{m}}$ during pre-CPB TEE was 4.0 to $9.3 \mathrm{mmHg}$ (95\% CI; $p<0.001)$ lower compared with preoperative TTE; (2) AVA during pre-CPB TEE was 0.04 to $0.15 \mathrm{~cm}^{2}(95 \% \mathrm{CI}$ $\mathrm{p}<0.001$ ) higher compared to preoperative TTE; (3) these 
Table 1. Patient Demographics

\begin{tabular}{|c|c|}
\hline Characteristic & $\mathrm{n}=92$ \\
\hline Age at surgery, years & $71.2 \pm 11.9$ \\
\hline Male sex, $\mathrm{n}(\%)^{\dagger}$ & $44(48)$ \\
\hline Body mass index, $\mathrm{kg} / \mathrm{m}^{2 *}$ & $29.9 \pm 6.2$ \\
\hline Time from TTE to pre-CPB TEE, days ${ }^{\ddagger}$ & $38.1 \pm 37.8$ \\
\hline \multicolumn{2}{|l|}{ Surgical procedure, $\mathrm{n}(\%)^{\dagger}$} \\
\hline AVR & $60(65)$ \\
\hline $\mathrm{CABG}+\mathrm{AVR}$ & $32(35)$ \\
\hline \multicolumn{2}{|l|}{ Aortic insufficiency, $\mathrm{n}(\%)^{\dagger}$} \\
\hline None/trace & $21(23)$ \\
\hline Mild & $34(37)$ \\
\hline Moderate & $28(30)$ \\
\hline \multicolumn{2}{|l|}{ Mitral regurgitation, $\mathrm{n}(\%)^{\dagger}$} \\
\hline None/trace & $74(80)$ \\
\hline Mild & $18(20)$ \\
\hline
\end{tabular}

Abbreviations: AVR, aortic valve replacement; CABG, coronary artery bypass grafting.

* Data are presented as mean \pm standard deviation.

tData are presented as frequency in the population.

$\ddagger \mathrm{n}=78$ for body mass index, due to missing data.

differences imparted an underestimation of AS severity by 1 grade during pre-CPB TEE $39.1 \%$ and $15.2 \%$ of the time, when using $\mathrm{PG}_{\mathrm{m}}$ and AVA, respectively; (4) 5.4\% ( $\left.\mathrm{n}=5 / 92\right)$ of patients were classified as experiencing mild AS by at least 1 grading parameter during pre-CPB TEE, even though the preoperative TTE exhibited moderate AS or greater for both grading parameters.

Recent American College of Cardiology/American Heart Association guidelines for management of patients with valvular heart disease provide an algorithm for AVR decision making. ${ }^{2}$ This algorithm uses measurements to grade severity and provides recommendations for AVR for AS. The Dopplerderived $\mathrm{PG}_{\mathrm{m}}$ or peak velocity are primary determinants of severity, whereas presence of symptoms, LVEF, and AVA by continuity equation are critical branch points along the decision tree. Also, patients undergoing cardiac surgery for another reason, who also experience moderate AS, have a class IIa indication for AVR. ${ }^{2}$

Grading AS accurately using $\mathrm{PG}_{\mathrm{m}}$ and AVA, however, can be confusing due to the issue of grading discordance, defined as a difference in grading assignment between parameters.

Table 2. Mean Values of Aortic Stenosis Grading Parameters in Patients Undergoing AVR \pm CABG

\begin{tabular}{cccc}
\hline & $\begin{array}{c}\text { Preoperative TTE } \\
(\mathrm{n}=92)\end{array}$ & $\begin{array}{c}\text { Pre-CPB TEE } \\
(\mathrm{n}=92)\end{array}$ & $\begin{array}{c}\text { Difference } \\
(\mathrm{n}=92)\end{array}$ \\
\hline $\begin{array}{c}\text { Average mean } \\
\text { gradient }\end{array}$ & $46.0 \pm 14.3$ & $39.4 \pm 14.1$ & $-6.6^{*}(\mathrm{p}<0.001)$ \\
$(\mathrm{mmHg} \pm \mathrm{SD})$ & & & \\
$\begin{array}{c}\text { Average aortic valve } \\
\text { area }\left(\mathrm{cm}^{2} \pm \mathrm{SD}\right)\end{array}$ & $0.73 \pm 0.24$ & $0.83 \pm 0.31$ & $0.10^{+}(\mathrm{p}<0.001)$ \\
\hline
\end{tabular}

Abbreviations: AVR, aortic valve replacement; CABG, coronary artery bypass grafting; CPB, cardiopulmonary bypass; SD, standard deviation; TEE, transesophageal echocardiography; TTE, transthoracic echocardiography.

$* 95 \% \mathrm{Cl},-4.0$ to $-9.3 \mathrm{mmHg} ; \mathrm{p}<0.001$.

$+95 \% \mathrm{Cl}, 0.04-0.15 \mathrm{~cm}^{2} ; \mathrm{p}<0.001$.
Table 3. Change in AS Severity Based on Imaging Context and Grading Parameter

\begin{tabular}{lcc}
\hline & \multicolumn{2}{c}{$\begin{array}{c}\text { AS Grading Parameter Using } \\
\text { Preoperative TTE }(\mathrm{n}=92)\end{array}$} \\
\cline { 2 - 3 } & $\begin{array}{c}\text { Mean Gradient } \\
\left(\mathrm{PG}_{\mathrm{m}}\right)\end{array}$ & $\begin{array}{c}\text { Aortic Valve Area } \\
\text { (AVA) }\end{array}$ \\
\hline Impact of pre-CPB TEE on AS grade & & \\
1 grade higher & $5(5.4)^{*}$ & $4(4.3)^{\dagger}$ \\
No change & $51(55.4)$ & $75(81.5)$ \\
1 grade lower & $36(39.1)^{\ddagger}$ & $13(14.1)^{\ddagger}$ \\
2 grades lower & $0(0.0)$ & $1(1.1)^{\S}$ \\
Any change & $41(44.5)^{\ddagger}$ & $17(18.5)^{\ddagger}$ \\
\hline
\end{tabular}

NOTE. Data are expressed as number (percentage).

$p$ values for comparison of grade change to no change using 2-sided exact tests for binomial proportions:

Abbreviations: AS, aortic stenosis; AVA, aortic valve area; CPB, cardiopulmonary bypass; $\mathrm{PG}_{\mathrm{m}}$, mean pressure gradient.

$$
\begin{aligned}
& * p=0.02 \\
& \dagger p=0.04 \\
& \ddagger p<0.0001 \\
& \S p=0.3 .
\end{aligned}
$$

Grading discordance may occur even in the setting of normal LVEF because of reductions in left ventricular (LV) stroke volume unrelated to $\mathrm{LV}$ systolic function. ${ }^{10-14} \mathrm{~A}$ significant reason for grading discordance has been attributed to the existence of a distinct subtype of aortic valve disease, lowflow AS with preserved EF, also known as "paradoxical AS," which can occur in patients with LV hypertrophy in the context of small LV size, right heart dysfunction, diastolic dysfunction, or moderate or severe mitral regurgitation. ${ }^{13,15-18}$

Likewise, grading discordance is greater during pre-CPB TEE compared with preoperative TTE, even when controlling for LVEF and mitral regurgitation. ${ }^{19}$ Approximately $51 \%$ of patients undergoing AVR or AVR with CABG exhibit discordance between $\mathrm{PG}_{\mathrm{m}}$ and AVA grading across all grades (mild, moderate, and severe) of AS during pre-CPB TEE. ${ }^{19}$ Therefore, general anesthesia with positive-pressure ventilation may predictably decrease LV stroke volume, thereby affecting AS assessment in a similar fashion to the other characteristics that lead to "paradoxical AS." The gradient drop quantified in this study when comparing pre-CPB TEE with preoperative TTE was consistent with such reasoning. Further prospective work is necessary to confirm the effect of general anesthesia on AS grading parameters and to standardize pre-CPB TEE assessment to accurately grade AS, should incidental AS arise.

Because quantification of AS with mean gradient is highly dependent on beam alignment, suboptimal probe alignment or differences in alignment between TTE and pre-CPB TEE in the same patient could lead to discrepancies. For example, discrepancies based on apical versus nonapical views using TTE affect the highest velocity or gradient obtained. ${ }^{20}$ Although the authors routinely used measurements from all viewing windows and recorded the highest value, it is unclear to what extent beam alignment, when transitioning from TTE to preCPB TEE, affected the values obtained.

There was a small, albeit statistically significant, difference between preoperative TTE and pre-CPB TEE AVA. AVA calculations notoriously are fraught with error and are 
dependent on accurate LVOT diameter measurements. ${ }^{21-23}$ Two-dimensional TTE LVOT measurements underestimate the true LVOT area, especially in patients with AS, who demonstrate less circular LVOTs. ${ }^{22}$ In addition, LVOT diameter measurements differ slightly between imaging modalities based on the different viewing windows, parasternal long axis and midesophageal long axis, for TTE and TEE, respectively. Shiran et al, for example, demonstrated that TTE underestimated LVOT diameter compared with TEE, which subsequently translated to a smaller AVA for TTE versus TEE. ${ }^{24}$

As with mean gradient measurement, AVA calculation is highly dependent on accurate beam alignment with the direction of blood flow. The smaller impact on grading between TTE and TEE when using AVA calculations versus $\mathrm{PG}_{\mathrm{m}}$ may be due to its integration of LV stroke volume, which could mitigate the hemodynamic changes imparted by anesthesia. Further work is necessary to delineate the impact of simultaneously moving from one clinical context and imaging modality to the next and to determine what is the best way to grade incidental AS, given the current practice of applying TTEderived guideline cutoffs to pre-CPB TEE.

\section{Limitations}

The retrospective nature of this study limited the conclusions due to possible selection bias. However, the database search was large, spanning more than 1 decade, in a highvolume cardiothoracic surgical center, and was part of a dedicated perioperative echocardiography and cardiology fellowship-training program. Both preoperative TTE and preCPB TEE acquisition have standard protocols for image acquisition and accuracy enhancement through offline reviews by echocardiographers performing secondary assessments. All performing and reviewing echocardiographers for pre-CPB TEE received NBE special competence certification for advanced perioperative TEE.

Although an exclusion criterion of 6 months between TTE and pre-CPB TEE was used for study inclusion, the mean time from TTE to pre-CPB TEE was only 38.1 days (median time = 28 days) (see Table 1). Ideally, TTE would be performed simultaneously or as closely as possible to the time of pre-CPB TEE. However, the primary finding of this study showed that the mean gradient obtained during pre-CPB was lower than the mean gradient recorded during preoperative TTE. AS disease progression over the time span between imaging techniques would cause the mean gradient to increase in the absence of a decrease in ventricular systolic function.

The largest limitation in this study was the inability to control for cardiac output, arterial compliance, blood pressure, and other hemodynamic parameters. The echocardiographic data were not linked to real-time hemodynamics in a fashion that could be practically gleaned from intraoperative anesthetic records. Nevertheless, the sample size was large enough to distribute this unpredictability randomly across the population. The discrepancies seen in this study might be remedied if hemodynamics were normalized to values obtained at the time of preoperative TTE. Such a possibility, however, highlights the fact that no current guidelines suggest that pre-CPB echocardiographers perform such a maneuver to grade AS intraoperatively. If changes in $\mathrm{PG}_{\mathrm{m}}$ and AVA between preoperative TTE and pre-CPB TEE are the result of easily predictable and modifiable hemodynamic changes brought about by general anesthesia, these findings illustrate the need for further work to quantify such changes to standardize preCPB TEE for accurate AS assessment.

\section{Clinical Relevance}

Finding modest changes in grading assessment during preCPB TEE should not prompt a change in surgical planning if an echocardiographer discovers that such differences conflict with known, quality preoperative TTE (or TEE)-based grading. However, some patients present to cardiac anesthesiologists with incomplete, lost, poor quality, or old cardiac evaluations. Therefore, comparing pre-CPB TEE with preoperative TTE grading parameters provides an important framework for echocardiographers who identify incidental or previously unrecognized AS during pre-CPB TEE.

In this study, patients undergoing AVR demonstrated moderate or greater AS by both parameters during preoperative TTE; meanwhile, $5.4 \%(n=5 / 92)$ of patients exhibited mild AS based on at least 1 grading parameter during pre-CPB TEE. In a patient undergoing CABG or mitral valve surgery, moderate $\mathrm{AS}$ is an indication of $\mathrm{AVR} .^{2}$ Therefore, artificial underestimation of AS severity using pre-CPB TEE can be problematic for a perioperative echocardiographer tasked with providing useful information to the surgical team in cases of incidental AS. These findings suggested that the effects of general anesthesia and positive-pressure ventilation during the pre-CPB period cannot be ignored during such conditions. These findings also called into question the commonly accepted superiority of $\mathrm{PG}_{\mathrm{m}}$ measurements versus AVA calculations for AS assessment during pre-CPB TEE because $\mathrm{PG}_{\mathrm{m}}$ differences between modalities affected a grade change more often than did AVA calculations. ${ }^{25}$

\section{CONCLUSIONS}

Rigid application of current guidelines and TTE-based AS grading cutoffs to pre-CPB TEE $\mathrm{PG}_{\mathrm{m}}$ and AVA values should be performed with caution in the setting of incidental AS. Alternatively, once precise mechanisms for discrepancies of $\mathrm{PG}_{\mathrm{m}}$ and AVA between preoperative TTE and pre-CPB TEE are clarified, hemodynamic alterations or mathematical conversions may be possible to accurately grade AS using AVA or $\mathrm{PG}_{\mathrm{m}}$ during pre-CPB TEE. Meanwhile, 2-dimensional qualitative examination and other grading parameters, such as indexed AVA or dimensionless index, should not be overlooked in the setting of low $\mathrm{PG}_{\mathrm{m}}$ during pre-CPB TEE AS assessment, given the potential confounding effects of general anesthesia. Further work is necessary to determine the best way to assign an AS grade during pre-CPB TEE should incidental AS arise. 


\section{REFERENCES}

1. Eltzschig HK, Rosenberger P, Loffler M, et al: Impact of intraoperative transesophageal echocardiography on surgical decisions in 12,566 patients undergoing cardiac surgery. Ann Thorac Surg 85: 845-852, 2008

2. Nishimura RA, Otto CM, Bonow RO, et al: 2014 AHA/ACC guideline for the management of patients with valvular heart disease: Executive summary: A report of the American College of Cardiology/ American Heart Association Task Force on Practice Guidelines. J Am Coll Cardiol 63:2438-2488, 2014

3. Otto CM, Pearlman AS, Comess KA, et al: Determination of the stenotic aortic valve area in adults using Doppler echocardiography. J Am Coll Cardiol 7:509-517, 1986

4. Oh JK, Taliercio CP, Holmes DR Jr, et al: Prediction of the severity of aortic stenosis by Doppler aortic valve area determination: Prospective Doppler-catheterization correlation in 100 patients. J Am Coll Cardiol 11:1227-1234, 1988

5. Galan A, Zoghbi WA, Quinones MA: Determination of severity of valvular aortic stenosis by Doppler echocardiography and relation of findings to clinical outcome and agreement with hemodynamic measurements determined at cardiac catheterization. Am J Cardiol 67: 1007-1012, 1991

6. Otto CM, Burwash IG, Legget ME, et al: Prospective study of asymptomatic valvular aortic stenosis. Clinical, echocardiographic, and exercise predictors of outcome. Circulation 95:2262-2270, 1997

7. Baumgartner $\mathrm{H}$, Hung $\mathrm{J}$, Bermejo $\mathrm{J}$, et al: Echocardiographic assessment of valve stenosis: EAE/ASE recommendations for clinical practice. Eur J Echocardiogr 10:1-25, 2009

8. Zoghbi WA, Farmer KL, Soto JG, et al: Accurate noninvasive quantification of stenotic aortic valve area by Doppler echocardiography. Circulation 73:452-459, 1986

9. Baumgartner H, Hung J, Bermejo J, et al: Echocardiographic assessment of valve stenosis: EAE/ASE recommendations for clinical practice. J Am Soc Echocardiogr 22:1-23: quiz 101-102, 2009

10. Jander N, Minners J, Holme I, et al: Outcome of patients with low-gradient "severe" aortic stenosis and preserved ejection fraction. Circulation 123:887-895, 2011

11. Minners J, Allgeier M, Gohlke-Baerwolf C, et al: Inconsistencies of echocardiographic criteria for the grading of aortic valve stenosis. Eur Heart J 29:1043-1048, 2008

12. Minners J, Allgeier M, Gohlke-Baerwolf $\mathrm{C}$, et al: Inconsistent grading of aortic valve stenosis by current guidelines: Haemodynamic studies in patients with apparently normal left ventricular function. Heart 96:1463-1468, 2010

13. Hachicha Z, Dumesnil JG, Bogaty P, et al: Paradoxical low-flow, low-gradient severe aortic stenosis despite preserved ejection fraction is associated with higher afterload and reduced survival. Circulation 115: 2856-2864, 2007

14. Barasch E, Fan D, Chukwu EO, et al: Severe isolated aortic stenosis with normal left ventricular systolic function and low transvalvular gradients: Pathophysiologic and prognostic insights. J Heart Valve Dis 17:81-88, 2008

15. Clavel MA, Pibarot P, Dumesnil JG: Paradoxical low flow aortic valve stenosis: Incidence, evaluation, and clinical significance. Curr Cardiol Rep 16:431, 2014

16. Tribouilloy C, Rusinaru D, Marechaux S, et al: Low-gradient, low-flow severe aortic stenosis with preserved left ventricular ejection fraction: Characteristics, outcome, and implications for surgery. J Am Coll Cardiol 65:55-66, 2015

17. Leong DP, Pizzale S, Haroun MJ, et al: Factors associated with low flow in aortic valve stenosis. J Am Soc Echocardiogr 29: 158-165, 2016

18. Pibarot P, Dumesnil JG: Low-flow, low-gradient aortic stenosis with normal and depressed left ventricular ejection fraction. J Am Coll Cardiol 60:1845-1853, 2012

19. Whitener G, McKenzie J, Akushevich I, et al: Discordance in grading methods of aortic stenosis by pre-cardiopulmonary bypass transesophageal echocardiography. Anesth Analg 122:953-958, 2016

20. Thaden JJ, Nkomo VT, Lee KJ, et al: Doppler imaging in aortic stenosis: The importance of the nonapical imaging windows to determine severity in a contemporary cohort. J Am Soc Echocardiogr 28:780-785, 2015

21. Mehrotra P, Flynn AW, Jansen K, et al: Differential left ventricular outflow tract remodeling and dynamics in aortic stenosis. J Am Soc Echocardiogr 28:1259-1266, 2015

22. Pibarot P, Clavel MA: Left ventricular outflow tract geometry and dynamics in aortic stenosis: Implications for the echocardiographic assessment of aortic valve area. J Am Soc Echocardiogr 28. 1267-1269, 2015

23. Kasel AM, Cassese S, Bleiziffer S, et al: Standardized imaging for aortic annular sizing: Implications for transcatheter valve selection. JACC Cardiovasc Imaging 6:249-262, 2013

24. Shiran A, Adawi S, Ganaeem M, et al: Accuracy and reproducibility of left ventricular outflow tract diameter measurement using transthoracic when compared with transesophageal echocardiography in systole and diastole. Eur J Echocardiogr 10:319-324, 2009

25. Skubas NJ, Shernan SK, Bollen B: An Overview of the American College of Cardiology/American Heart Association 2014 Valve Heart Disease Practice Guidelines: What is its relevance for the anesthesiologist and perioperative medicine physician? Anesth Analg 121:1132-1138, 2015 